\title{
Hydrogen ameliorates oxidative stress via PI3K-Akt signaling pathway in UVB-induced HaCaT cells
}

\author{
BINGXIN ZHANG ${ }^{1 *}$, ZISHEN ZHAO $^{2 *}$, XIAOYIN MENG ${ }^{3}$, HONGGUANG CHEN $^{4}$, \\ GUOJUN FU ${ }^{2}$ and KELIANG XIE ${ }^{4}$ \\ ${ }^{1}$ Department of Dermatology, First Teaching Hospital of Tianjin University of Traditional Chinese Medicine, Tianjin 300193; \\ ${ }^{2}$ Department of Dermatology, Cangzhou City People's Hospital, Cangzhou, Hebei 061000; \\ ${ }^{3}$ Department of Gynaecology and Obstetrics, Tianjin Hospital, Tianjin 300211; \\ ${ }^{4}$ Department of Anesthesiology, Tianjin Institute of Anesthesiology, \\ General Hospital of Tianjin Medical University, \\ Tianjin 300052, P.R. China
}

Received August 11, 2017; Accepted March 1, 2018

DOI: $10.3892 / \mathrm{ijmm} .2018 .3550$

\begin{abstract}
Chronic ultraviolet (UV) exposure-induced oxidative stress is associated with the pathogenesis of skin damage. However, the nuclear factor erythroid-2-related factor 2 (Nrf2) pathway is a critical factor in protecting cells against UVB-induced injury through inhibiting oxidative stress. Furthermore, Nrf2 activation requires the involvement of the phosphoinositide-3 kinase (PI3K)/protein kinase B (AKT) pathway, which has a major role in survival of various cell types. Molecular hydrogen exerts protective effects on UV-induced injury, but the underlying mechanisms have remained elusive. The present study assessed the protective effects of hydrogen against oxidative stress-induced injury caused by UVB irradiation and investigated the molecular mechanisms. In vitro, UVB-induced HaCaT cells were collected for the detection of reactive oxygen species, 8 -iso-prostaglandin F2 $\alpha$, malondialdehyde via fluorescence spectrometry and ELISA; cell activity and cytotoxicity by MTT and lactate dehydrogenase assays, respectively. Additionally, the expression level of PI3K, Akt, Nrf2 and heme oxygenase-1 (HO-1) were investigated using western blot, etc. All of the results indicated that hydrogen decreased the levels of reactive oxygen species, 8-iso-prostaglandin F2 $\alpha$ and malondialdehyde, and promoted the UVB exposure-induced expression of PI3K, Akt, Nrf2 and heme
\end{abstract}

Correspondence to: Dr Bingxin Zhang, Department of Dermatology, First Teaching Hospital of Tianjin University of Traditional Chinese Medicine, 314, Anshan Road, Tianjin 300193, P.R. China

E-mail: zbingxin@163.com

*Contributed equally

Key words: oxidative stress, ultraviolet, hydrogen, nuclear factor erythroid-2-related factor 2, phosphoinositide-3 kinase oxygenase- 1 in $\mathrm{HaCaT}$ cells. Of note, PI3K inhibition partially reversed the effects of hydrogen on UVB-induced $\mathrm{HaCaT}$ cells. Therefore, hydrogen effectively protects cells from UVB radiation-induced oxidative stress by inhibiting $\mathrm{Nrf} 2 / \mathrm{HO}-1$ activation through the PI3K/Akt signaling pathway.

\section{Introduction}

The skin is a primary of protective organ system and guards against destructive stimuli, including solar ultraviolet (UV) radiation. With ozone layer depletion through halogen-containing compound emissions caused by human activities, large amounts of UV radiation are able to reach to the earth, which increases the level of UV radiation. Once absorbed by human skin, severe skin problems, including cutaneous malignancies then arise (1). After sun exposure, specific pathophysiological insults, including massive elastosis, collagen degeneration, pigmentation, sallowness and deep wrinkling, occur in response to UV $(2,3)$. Solar UV radiation on the surface of the earth consists of 90-99\% UVA and 1-10\% UVB rays (4), which are classified as wavelength ranges of 315-400 and 280-315 $\mathrm{nm}$, respectively. While UVB radiation constitutes $1-10 \%$ of the UV energy that reaches the surface of the earth, it is 1,000-10,000-fold more carcinogenic than UVA radiation, which makes up 90-99\% of UV energy (5).

Excessive exposure to UV radiation predisposes humans to unhealthy conditions, including sunburn, inflammation, oxidative stress, DNA damage, solar erythema, premature aging, suppression of the immune system and skin cancers (6-9). Of note, exposure to UVB radiation induces excessive generation of reactive oxygen species (ROS), including hydrogen peroxide, superoxide anions, hydroxyl radicals and singlet oxygen (10), which may oxidize and damage cellular lipids, proteins and DNA, leading to pathophysiological changes and even skin aging $(11,12)$. Accumulative studies have indicated that the direct absorption of UVB photons, which underlies UVB-induced oxidative injury, leads to the formation of oxidative products $(13,14)$. In addition, UVB influences the 
expression of anti-oxidant enzymes, activates the anti-oxidant defense system and ultimately interferes with the ability of the skin to protect itself against ROS and reactive nitrogen species (15).

Nuclear factor erythroid-2-related factor $2(\mathrm{Nrf} 2)$ is a crucial anti-oxidant signaling molecule in mammalian cells. Of note, the Nrf2/antioxidant response element (ARE) signaling pathway has a vital protective role in UV radiation-induced skin cell injury, which results from the induction of DNA damage or apoptosis (16-18). After exposure to UV, Nrf2 dissociates from Kelch-like ECH-associated protein 1 (Keap1) to translocate into the nucleus and combine with AREs. The Nrf2/ARE signaling pathway is thereby activated, which further activates downstream cytoprotective genes and upregulates anti-oxidant enzymes, including heme oxygenase-1 (HO-1), to counteract UVB-mediated cellular injury and eventually maintain cell survival (19). Zhong and Li (20) revealed that SKPs have a protective role in resisting UVB-induced dermal fibroblast cell damage and aging via upregulation of Nrf2. Furthermore, the phosphatidylinositol 3-kinase (PI3K)/Akt pathway may also improve cell survival and is required for Nrf2 activation (21). Activation of the Nrf2 signaling pathway requires the involvement of PI3K phosphorylation and downstream Akt phosphorylation (22-25). Activation of Nrf2/ARE via PI3K signaling has a protective effect on skin cells against UV-induced DNA damage (26).

Numerous studies have verified that hydrogen exerts protective anti-oxidant and anti-inflammatory effects, and effectively scavenges ROS to promote a protective system in cells and organs. It was also revealed that hydrogen protects cells and tissues from oxidative injury by activating Nrf2/HO-1 (27) and the Akt signaling pathway $(28,29)$. In addition, hydrogen was also reported to protect the skin from psoriasis (30), dermatitis induced by local radiation (31) and oxidative damage induced by high glucose or mannitol (32). Previous studies by our group have reported that hydrogen alleviates UVB-induced inflammation $(33,34)$ and oxidative damage $(35)$.

Although hydrogen mitigates inflammation and oxidative damage in skin-associated diseases, the detailed mechanisms of the effects of hydrogen in UVB-radiated HaCaT cells has remained elusive. The present study first assessed the effects of hydrogen on oxidative damage in $\mathrm{HaCaT}$ cells irradiated with UVB and subsequently aimed to examine the mechanistic involvement of the regulatory effects of the PI3K/Akt/Nrf2 signaling pathway.

\section{Materials and methods}

Cell culture and UVB radiation. The human keratinocyte cell line $\mathrm{HaCaT}$ was purchased from the American Type Culture Collection (Manassas, VA, USA) and cultured in Dulbecco's modified of Eagle's medium (DMEM) containing $10 \%$ heat-inactivated fetal bovine serum (FBS; Thermo Fisher Scientific, Inc., Waltham, MA, USA), $100 \mathrm{U} / \mathrm{ml}$ penicillin and $100 \mu \mathrm{g} / \mathrm{ml}$ streptomycin in a humidified atmosphere containing $5 \% \mathrm{CO}_{2}$ at $37^{\circ} \mathrm{C}$. HaCaT cells were seeded $1 \times 10^{6}$ cells $/ \mathrm{ml}$ in 6-well plates and allowed to adhere for $24 \mathrm{~h}$. Cells were exposed to UVB with an energy spectrum of UVB radiation $\left(1,10\right.$ or $\left.50 \mathrm{~mJ} / \mathrm{cm}^{2}\right)$ with a thin layer of PBS using a UVB lamp with a peak emission at $301 \mathrm{~nm}$ (280-320 nm; Sigma-Aldrich;
Merck KGaA, Darmstadt, Germany). After UVB irradiation for $30 \mathrm{sec}$, the cells were washed with warm PBS and incubated with serum-free DMEM for $24 \mathrm{~h}$. LY294002 (30 $\mu \mathrm{M}$; Sigma-Aldrich; Merck KGaA) was added to the medium $2 \mathrm{~h}$ prior UVB irradiation. Cells in the control group underwent the same schedule for medium changes without UVB irradiation.

Hydrogen treatment. A previously described method was used with certain modifications (36). In brief, hydrogen was dissolved in DMEM supplemented with 10\% FBS for $4 \mathrm{~h}$ under high pressure $(0.4 \mathrm{MPa})$ to reach a supersaturated level. The saturated hydrogen-rich medium was stored with no air in the vessel at $4^{\circ} \mathrm{C}$ under atmospheric pressure in an aluminum bag. Hydrogen-rich medium was freshly prepared every week to ensure that a concentration of $0.6 \mathrm{mmol} / 1$ hydrogen was maintained. A needle-type $\mathrm{H}_{2}$ sensor (Unisense A/S, Aarhus, Denmark) was used to measure the hydrogen concentration of the media according to the method published by Chen et al (27).

MTT assay. Cells were seeded into a 96-well plate at a density of 1,000 cells/well. Different treatments were completed as per the experimental schedule. The cells were washed with PBS, the medium was removed from each well, and the cells were incubated with $200 \mu \mathrm{l}$ of MTT $(0.5 \mathrm{mg} / \mathrm{ml})$ for $4 \mathrm{~h}$ at $37^{\circ} \mathrm{C}$. The formazan crystals that had formed were dissolved with $150 \mu \mathrm{l}$ dimethylsulfoxide after the medium was removed. The plates were incubated on an agitator for $15 \mathrm{~min}$ at room temperature. The absorbance of each well was measured at $490 \mathrm{~nm}$ using a microplate reader (Bio-Rad Laboratories, Inc., Hercules, CA, USA). The experiment was repeated five times.

Lactate dehydrogenase ( $\mathrm{LDH}$ ) detection. Cells were seeded at a density of $1 \times 10^{5}$ cells/well in a 12-well plate and incubated at $37^{\circ} \mathrm{C}$ with $5 \% \mathrm{CO}_{2}$ for $24 \mathrm{~h}$. Different treatments were completed as per the experimental schedule. Subsequently, the supernatant from each well was collected and the cytolytic activity was measured with an LDH cytotoxicity detection kit (Nanjing Jiancheng, Nanjing, China). The LDH levels were expressed as percentages of the LDH levels observed in the control cultures.

Intracellular ROS detection. Intracellular ROS levels were measured with a dichlorodihydrofluorescein diacetate (DCFH-DA) assay. HaCaT cells, at a density of $1 \times 10^{4}$ cells/well, were seeded in a 96-well plate, cultured for $6 \mathrm{~h}$ and treated according to the indicated experimental schedules for $24 \mathrm{~h}$. A total of $25 \mu \mathrm{M}$ DCFH-DA was added to the medium, followed by incubation at $37^{\circ} \mathrm{C}$ for $30 \mathrm{~min}$ in the dark. The fluorescence of the 2',7'-dichlorofluorescein product was detected with a PerkinElmer LS-5B fluorescence spectrometer (PerkinElmer, Waltham, MA, USA). The amounts of ROS were expressed as percentages of the ROS observed in the control cultures. All experiments for the standards and samples were performed in duplicate.

8-iso-prostaglandin F2 $\alpha$ (8-iso PGF2 $\alpha$ ) and malondialdehyde (MDA) detection. The culture media were collected and centrifuged at $1,000 \mathrm{x} \mathrm{g}$ for $10 \mathrm{~min}$ at $4^{\circ} \mathrm{C}$, and the supernatants were collected for detection. To estimate oxidative stress, 
A

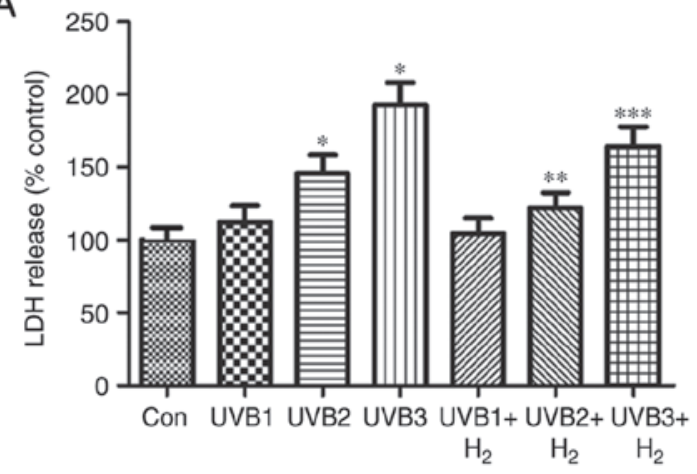

$\mathrm{B}$

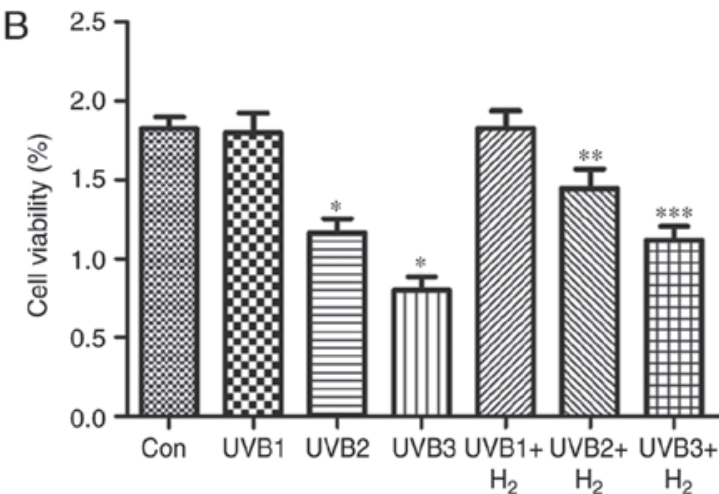

Figure 1. Effect of hydrogen on LDH release and cell proliferation induced by different doses of UVB radiation. HaCaT cells were irradiated with UVB at 1,10 and $50 \mathrm{~mJ} / \mathrm{cm}^{2}$ for $30 \mathrm{sec}$. In groups $\mathrm{UVB} 1+\mathrm{H}_{2}, \mathrm{UVB} 2+\mathrm{H}_{2}$ and $\mathrm{UVB} 3+\mathrm{H}_{2}$, normal medium was replaced with hydrogen-rich medium, and cells were further cultured for $24 \mathrm{~h}$. Supernatant was collected for (A) detection of LDH and cells were subjected to an (B) MTT assay. Values are expressed as the mean \pm standard error of the mean $(n=6)$. ${ }^{*} \mathrm{P}<0.05$ compared with Con group; ${ }^{* * *} \mathrm{P}<0.05$ compared with UVB2 group; ${ }^{* * * *} \mathrm{P}<0.05$ compared with UVB3 group. UVB1/2/3, UVB at 1,10 and $50 \mathrm{~mJ} / \mathrm{cm}^{2}$. LDH, lactate dehydrogenase; UVB, ultraviolet B; Con, control

8-iso-prostaglandin F2 $\alpha$ (8-iso PGF2 $\alpha$ ) was measured with a kit (cat. no. 316350; Cayman Chemical Company, Ann Arbor, MI, USA) using a microplate reader (CA 94089; Molecular Devices, Sunnyvale, CA, USA) according to the manufacturer's recommendations. For MDA detection, supernatants were processed using the kit (cat. no. 10009202-1; Cayman Chemical Company) as per the manufacturer's protocols. All experiments for the standards and samples were performed in duplicate.

Western blot analysis. After the incubations were completed, the cells were lysed with lysis buffer on ice for $10 \mathrm{~min}$ in radioimmunoprecipitation assay lysis buffer, and the protein was thereby extracted. The lysates were then collected and the protein concentration was evaluated via a Bicinchoninic Acid protein assay kit (Thermo Fisher Scientific, Inc.). The concentration of all lysates was adjusted to be the same according to the results of the BCA assay. Proteins (30 $\mu \mathrm{g}$ per lane) were separated by $12 \%$ SDS-PAGE and transferred onto polyvinylidene fluoride membranes (Immobilon ${ }^{\circledR}$-P Transfer Membrane; EMD Millipore, Billerica, MA, USA). Nuclear extracts for Nrf2 immunoblotting were prepared with the NE-PER nuclear kit (Pierce; Thermo Fisher Scientific, Inc.). The membranes were blocked with 5\% non-fat milk in Tris-HCL-based buffered saline with $0.1 \%$ Tween-20 (Sigma-Aldrich; Merck KGaA) at $\mathrm{pH} 7.4$ for $1 \mathrm{~h}$ and incubated with the following primary antibodies overnight at $4^{\circ} \mathrm{C}$ : $\beta$-actin $(1: 2,000$; cat. no. ab8226), Nrf2 (1:1,000; cat. no. ab62352), HO-1 (1:1,000; cat. no. ab13243), PI3K (1:1,000; cat. no. ab182651), phosphorylated (p)-PI3K (1:500 dilution; cat. no. ab182651), Akt (1:500 dilution; cat. no. ab18785) and p-Akt (1:500; cat. no. ab38449) (Abcam, Cambridge, MA, USA). The membranes were then incubated with corresponding horseradish peroxidase-conjugated secondary antibodies (anti-mouse, sc-2005; 1:5,000; Santa Cruz Biotechnology, Inc., Dallas, TX, USA and anti-rabbit, sc-2357, 1:5,000; Santa Cruz Biotechnology, Inc.). Protein bands were detected with a ChemiDoc ${ }^{\text {TM }}$ (Bio-Rad Laboratories, Inc., Hercules, CA, USA). $\beta$-actin expression was used as the reference. The protein expression level was quantified with Quantity One version 4.5.2 software (Bio-Rad Laboratories, Inc.)
Statistical analysis. Values are expressed as the mean \pm standard error of the mean. Multigroup comparisons were evaluated using one-way analysis of variance followed by Tukey's post hoc test using GraphPad Prism version 5 software (GraphPad Inc., La Jolla, CA, USA). P $<0.05$ was considered to indicate a statistically significant difference.

\section{Results}

Effects of different doses of $U V B$ radiation on $L D H$ release and cell viability. UVB radiation disrupted the cells, decreased cell viability and increased LDH release. The LDH leakage assay is based on the measurement of LDH activity in the extracellular medium. In the present study, various doses of UVB radiation were used to imitate the effects of UVB on human keratinocytes. Irradiation of HaCaT cells with 1,10 and $50 \mathrm{~mJ} / \mathrm{cm}^{2} \mathrm{UVB}$ had different effects on LDH release and inhibited cell viability, but only 10 and $50 \mathrm{~mJ} / \mathrm{cm}^{2} \mathrm{UVB}$ had significant effects compared with the control group $(\mathrm{P}<0.05$; Fig. 1A and B). Furthermore, it was investigated whether hydrogen exerts a protective effect on $\mathrm{HaCaT}$ cells irradiated by UVB. Hydrogen treatment reduced the LDH release of cells after exposure to 1,10 and $50 \mathrm{~mJ} / \mathrm{cm}^{2} \mathrm{UVB}$, which was significant at 10 and $50 \mathrm{~mJ} / \mathrm{cm}^{2}$ UVB (P<0.05; Fig. 1A). The results of the cell viability assay were similar to those of the LDH assay, namely that 10 and $50 \mathrm{~mJ} / \mathrm{cm}^{2}$ UVB significantly reduced the cell viability compared with that in the control group, while hydrogen significantly promoted the cell viability after exposure to 10 and $50 \mathrm{~mJ} / \mathrm{cm}^{2} \mathrm{UVB}(\mathrm{P}<0.05$; Fig. 1B).

Hydrogen treatment alleviates ROS, 8-iso PGF $2 \alpha$ and $M D A$ release in $U V B$-induced HaCaT cells. UV radiation is associated with the formation of ROS, leading to skin aging and photocarcinogenesis. To determine whether hydrogen alleviated UVB-mediated ROS accumulation and oxidative products, ROS, 8 -iso PGF $2 \alpha$ and MDA release levels caused by the augmentation of the endogenous anti-oxidant capacity was measured in UVB-induced $\mathrm{HaCaT}$ cells. The UVB-induced intracellular oxidative stress level was measured with a redox-sensitive dye, DCFH-DA. The results indicated that UVB radiation stimulated ROS 

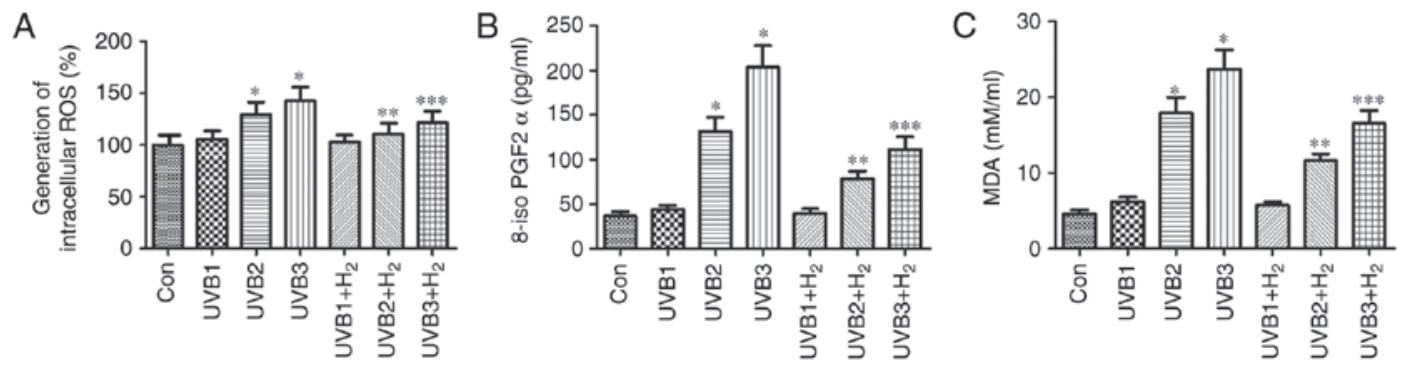

Figure 2. Effect of hydrogen on (A) ROS, (B) 8-iso PGF2 $\alpha$ and (C) MDA induced by various doses of UVB radiation. HaCaT cells were irradiated with UVB at 1,10 and $50 \mathrm{~mJ} / \mathrm{cm}^{2}$ for $30 \mathrm{sec}$. In groups UVB1+ $\mathrm{H}_{2}, \mathrm{UVB} 2+\mathrm{H}_{2}$ and $\mathrm{UVB} 3+\mathrm{H}_{2}$, normal medium was replaced with hydrogen-rich medium, and cells were further cultured for $24 \mathrm{~h}$. Supernatant was collected for detection of ROS and 8-iso PGF2 $\alpha$ and MDA. Values are expressed as the mean \pm standard error of the mean $(\mathrm{n}=6)$. " $\mathrm{P}<0.05$ compared with Con group; ${ }^{* *} \mathrm{P}<0.05$ compared with UVB2 group; ${ }^{* * * *} \mathrm{P}<0.05$ compared with UVB3 group. UVB1/2/3, UVB at 1,10 and $50 \mathrm{~mJ} / \mathrm{cm}^{2}$. UVB, ultraviolet B; Con, control; ROS, reactive oxygen species; MDA, malondialdehyde; 8-iso PGF2 $\alpha, 8$-iso-prostaglandin F2 $\alpha$.
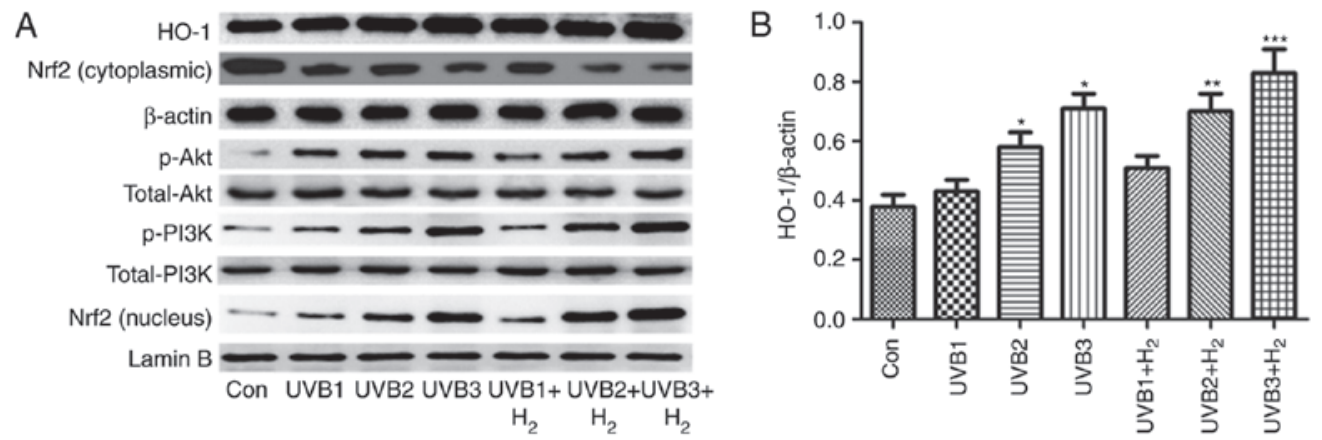

C

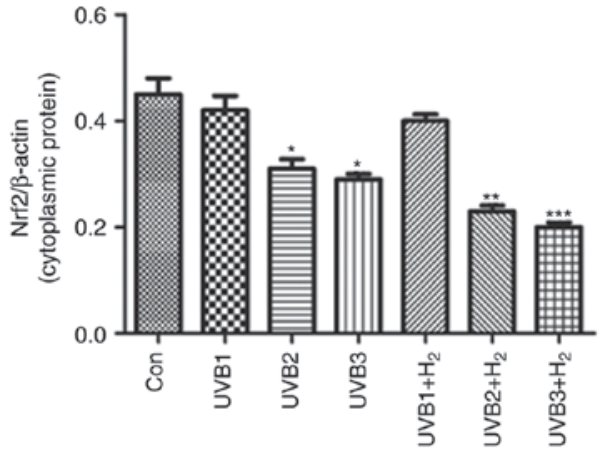

$\mathrm{E}$

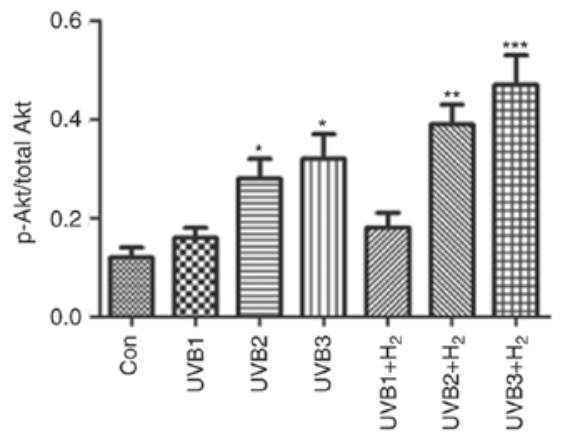

D

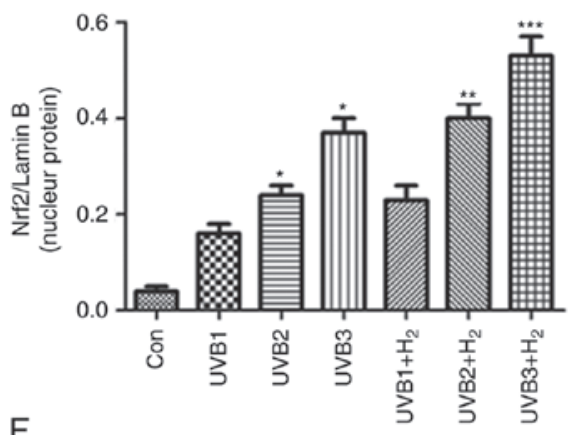

$\mathrm{F}$

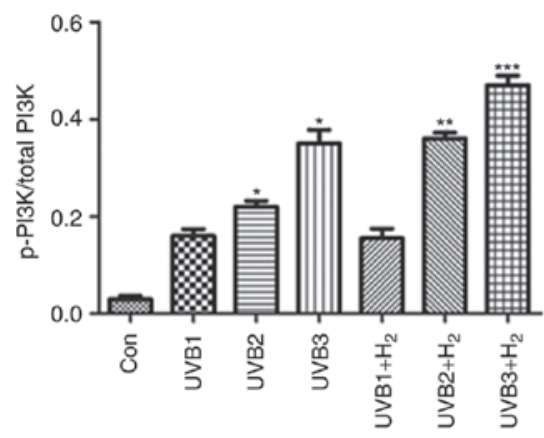

Figure 3. Nrf2, HO-1, Akt and PI3K expression after hydrogen and UVB treatment of HaCaT cells. HaCaT cells were irradiated with UVB at 1, 10 and $50 \mathrm{~mJ} / \mathrm{cm}^{2}$ for $30 \mathrm{sec}$. In groups $\mathrm{UVB} 1+\mathrm{H}_{2}, \mathrm{UVB} 2+\mathrm{H}_{2}$ and $\mathrm{UVB} 3+\mathrm{H}_{2}$, normal medium was replaced with hydrogen-rich medium, and cells were further cultured for $24 \mathrm{~h}$. Cells were collected and subjected to western blot analysis. (A) Representative western blot image. (B-F) Quantified expression levels of (B) HO-1, (C) cytosolic Nrf2, (D) nuclear Nrf2, (E) p/total Akt and (F) p/total PI3K. Values are expressed as the mean \pm standard error of the mean (n=6). ${ }^{*} \mathrm{P}<0.05$ compared with Con group; ${ }^{* *} \mathrm{P}<0.05$ compared with UVB2 group; ${ }^{* * * *} \mathrm{P}<0.05$ compared with UVB3 group. UVB, ultraviolet B; Con, control; p-PI3K, phosphorylated phosphoinositide-3 kinase; HO-1, heme oxygenase 1; Nrf2, nuclear factor erythroid-2-related factor 2.

production in a dose-dependent manner (Fig. 2A). Of note, hydrogen treatment obviously decreased the levels of ROS in UVB-irradiated cells ( $\mathrm{P}<0.05$; Fig. 2A). Similarly, 8-iso PGF2 $\alpha$ and MDA were also induced by UVB radiation in a dose-dependent manner compared with the control group; in addition, hydrogen significantly reduced the excessive 8 -iso PGF2 $\alpha$ and MDA release after UV irradiation ( $\mathrm{P}<0.05$; Fig. $2 \mathrm{~B}$ and $\mathrm{C}$ ). These results indicated that hydrogen alleviated ROS and their oxidative products induced by UVB irradiation. 

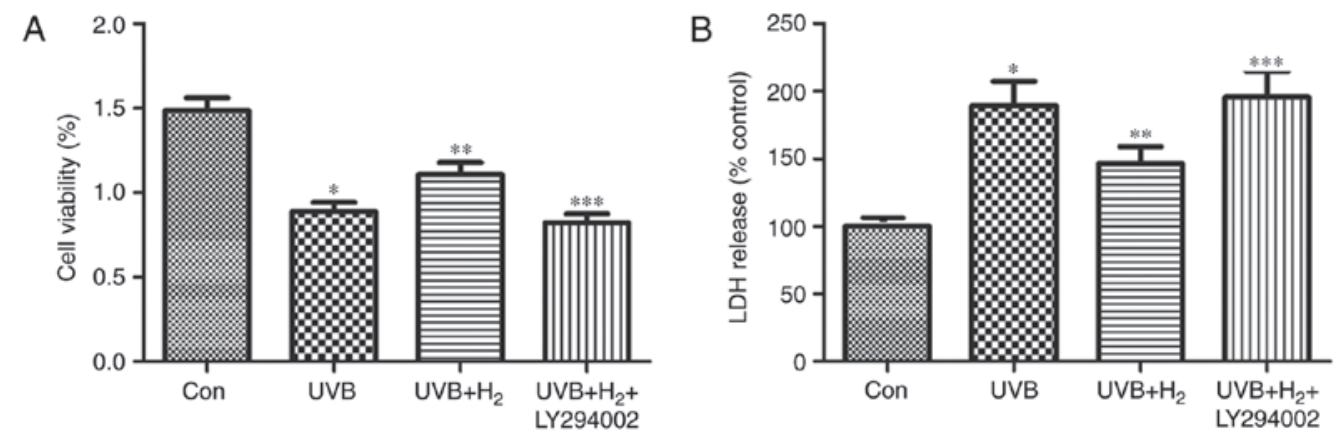

Figure 4. Role of PI3K in the protection of hydrogen against the cytotoxic and anti-proliferative effect of UVB on HaCaT cells. Cells were irradiated with $\mathrm{UVB}$ at $50 \mathrm{~mJ} / \mathrm{cm}^{2}$ for $30 \mathrm{sec}$. In group $\mathrm{UVB}+\mathrm{H}_{2}$, normal medium was replaced with hydrogen-rich medium and cells were further cultured for $24 \mathrm{~h}$. PI3K inhibitor LY294002 was used to inhibit PI3K at $24 \mathrm{~h}$ prior to UVB exposure. Supernatant and cells were utilized for detection of (A) LDH and (B) MTT assay, respectively. Values are expressed as the mean \pm standard error of the mean $(\mathrm{n}=6)$. ${ }^{*} \mathrm{P}<0.05$ compared with Con group; ${ }^{* *} \mathrm{P}<0.05$ compared with $\mathrm{UV} 2$ group; ${ }_{* * * *}^{*}<0.05$ compared with $\mathrm{UVB}+\mathrm{H}_{2}$ group. $\mathrm{PI} 3 \mathrm{~K}$, phosphoinositide-3 kinase; UVB, ultraviolet B; Con, control; LDH, lactate dehydrogenase.

Hydrogen increases the protein levels of p-PI3K, p-Akt and Nrf2 in UVB-irradiated HaCaT cells. Hydrogen reduced ROS and oxidation product levels in UVB-irradiated HaCaT cells. The Nrf2 pathway is an endogenous anti-oxidant system that protects cells from oxidative injury. The present study assessed whether hydrogen was able to activate the Nrf2 pathway to reduce ROS and oxidative products. The expression levels of $\mathrm{Nrf} 2$ and its downstream protein HO-1 were determined to confirm the role of Nrf2 in hydrogen-mediated protection (Fig. 3). UVB radiation increased Nrf2 expression in the nucleus in a concentration-dependent manner, while cytoplasmic Nrf2 expression was reduced $(\mathrm{P}<0.05$; Fig. 3A, C and D). In addition, the changes in HO-1 protein expression in the cytoplasm were similar with $\mathrm{Nrf} 2$ expression in the nucleus after UVB-radiation $(\mathrm{P}<0.05$; Fig. $3 \mathrm{~A}$ and $\mathrm{B})$. Hydrogen treatment clearly increased nuclear Nrf2 expression and HO-1 expression after UVB irradiation $(\mathrm{P}<0.05$; Fig. 3A-C).

PI3K is the upstream factor of the Nrf2 signaling pathway (37). To determine the effect of PI3K on the hydrogen-activated Nrf2 pathway in UVB-irradiated $\mathrm{HaCaT}$ cells, the protein levels of PI3K and its downstream effector, Akt, were measured by western blot analysis (Fig. 3). It was indicated that the p-Akt and p-PI3K levels were increased by UVB radiation compared with those in the control group $(\mathrm{P}<0.05$; Fig. 3A, E and F). Hydrogen further increased the p-Akt and p-PI3K levels in UVB-irradiated cells $(\mathrm{P}<0.05$; Fig. 3A, E and F). These results indicated that hydrogen increased the expression of PI3K/Akt and Nrf2/HO-1 pathway modulators.

Hydrogen exerts its protective effects against $U V$-induced cell damage through the PI3K/Akt pathway. To confirm that the effect of hydrogen on LDH release and cell viability was mediated through the PI3K/Akt pathway, cells were treated with a PI3K-specific inhibitor,LY294002, and its impact on the effects of hydrogen on LDH release and viability of irradiated cells was measured. Compared with the UVB group, the cell the viability increased and $\mathrm{LDH}$ release decreased in the $\mathrm{UVB}+\mathrm{H}_{2}$ group; however, following PI3K inhibition, it appeared that the protective roles exhibited by hydrogen on cell viability and damage were reduced $(\mathrm{P}<0.05$; Fig. $4 \mathrm{~A}$ and $\mathrm{B})$. These results

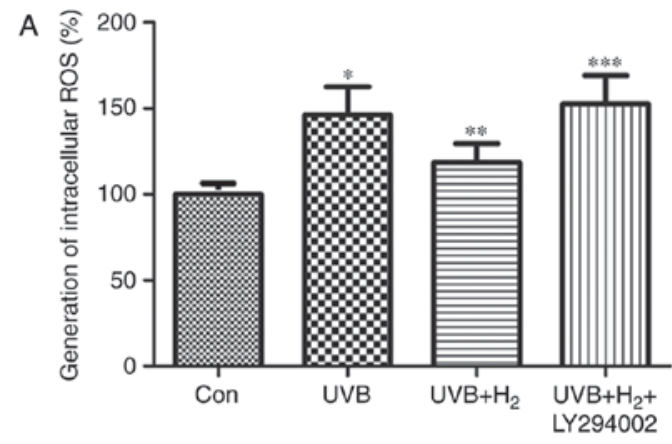

B
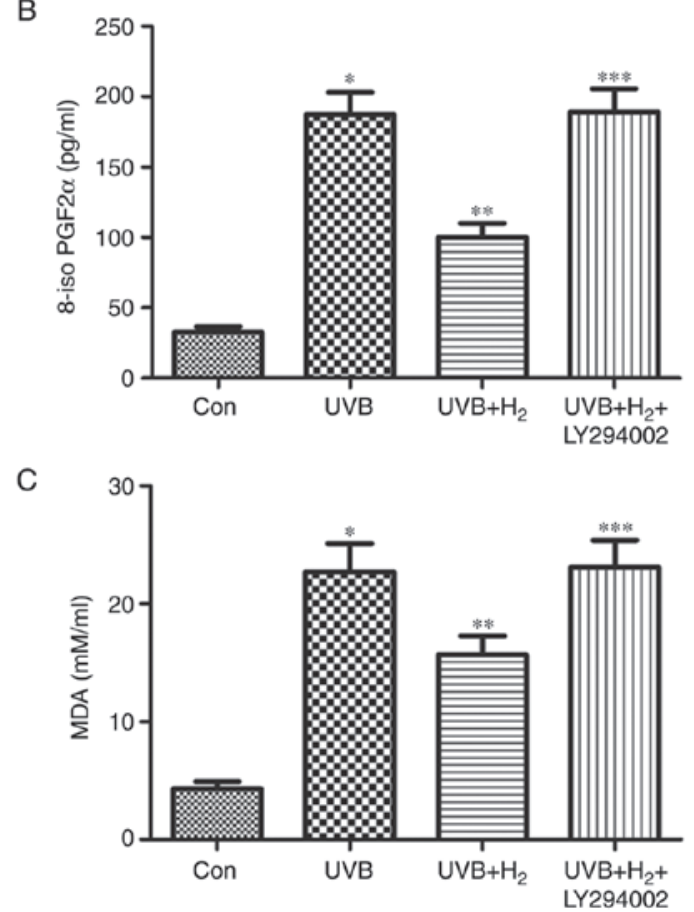

Figure 5. Role of PI3K in the protective effects of hydrogen against UVB-induced production of ROS, 8-iso PGF2 $\alpha$ and MDA by HaCaT cells. Cells were irradiated with UVB at $50 \mathrm{~mJ} / \mathrm{cm}^{2}$ for $30 \mathrm{sec}$. In group $\mathrm{UVB}+\mathrm{H}_{2}$, normal medium was replaced with hydrogen-rich medium and cells were further cultured for $24 \mathrm{~h}$. PI3K inhibitor LY294002 was used to inhibit PI3K at $24 \mathrm{~h}$ prior to UVB exposure. Supernatant were collected for detection of (A) ROS, (B) 8-iso PGF2 $\alpha$ and (C) MDA. Values are expressed as the mean \pm standard error of the mean $(n=6)$. * $P<0.05$ compared with Con group; ${ }^{* *} \mathrm{P}<0.05$ compared with UV2 group; ${ }^{* * *} \mathrm{P}<0.05$ compared with $\mathrm{UVB}+\mathrm{H}_{2}$ group. ROS, reactive oxygen species; MDA, malondialdehyde; 8-iso PGF2 $\alpha$, 8 -iso-prostaglandin F2 $\alpha$; PI3K, phosphoinositide-3 kinase; UVB, ultraviolet B; Con, control. 

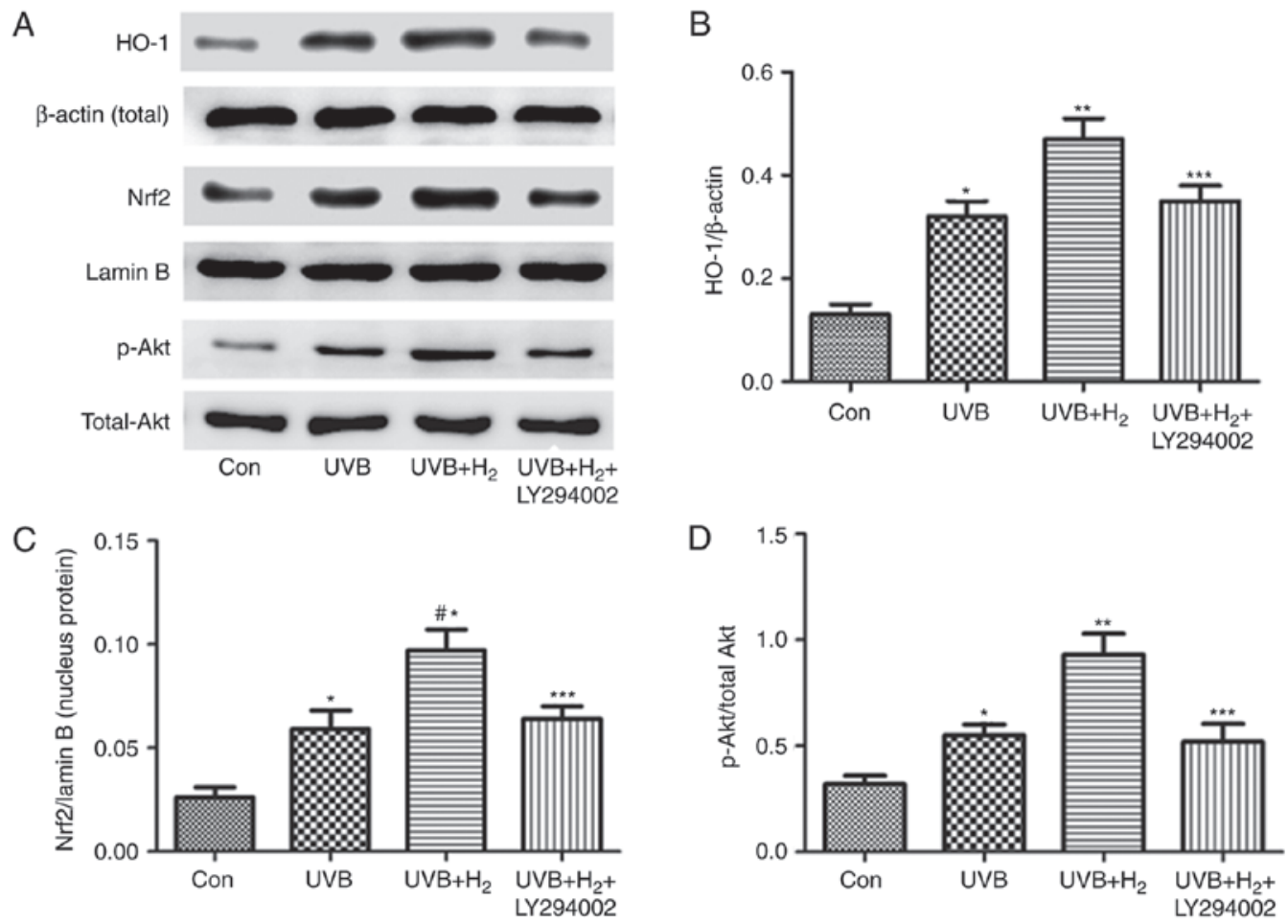

Figure 6. Role of PI3K on the effect of hydrogen on Nrf2, HO-1 and Akt levels in HaCaT cells induced by UVB radiation. Cells were irradiated with UVB at $50 \mathrm{~mJ} / \mathrm{cm}^{2}$ for $30 \mathrm{sec}$. In group $\mathrm{UVB}+\mathrm{H}_{2}$, normal medium was replaced with hydrogen-rich medium and cells were further cultured for $24 \mathrm{~h}$. PI3K inhibitor LY294002 was used to inhibit PI3K at $24 \mathrm{~h}$ prior to UVB exposure. Cells were collected for detection of Nrf2, HO-1 and p/total Akt by western blot. (A) Representative western blot image. (B-D) Quantified expression levels of (B) HO-1, (C) nuclear Nrf2 and (D) p/total Akt. Values are expressed as the mean \pm standard error of the mean $(n=6)$. " $\mathrm{P}<0.05$ compared with Con group; ${ }^{* *} \mathrm{P}<0.05$ compared with UV2 group; ${ }^{* * * * *} \mathrm{P}<0.05$ compared with $\mathrm{UVB}+\mathrm{H}_{2}$ group. UVB, ultraviolet B; Con, control; p-PI3K, phosphorylated phosphoinositide-3 kinase; HO-1, heme oxygenase 1; Nrf2, nuclear factor erythroid-2-related factor 2.

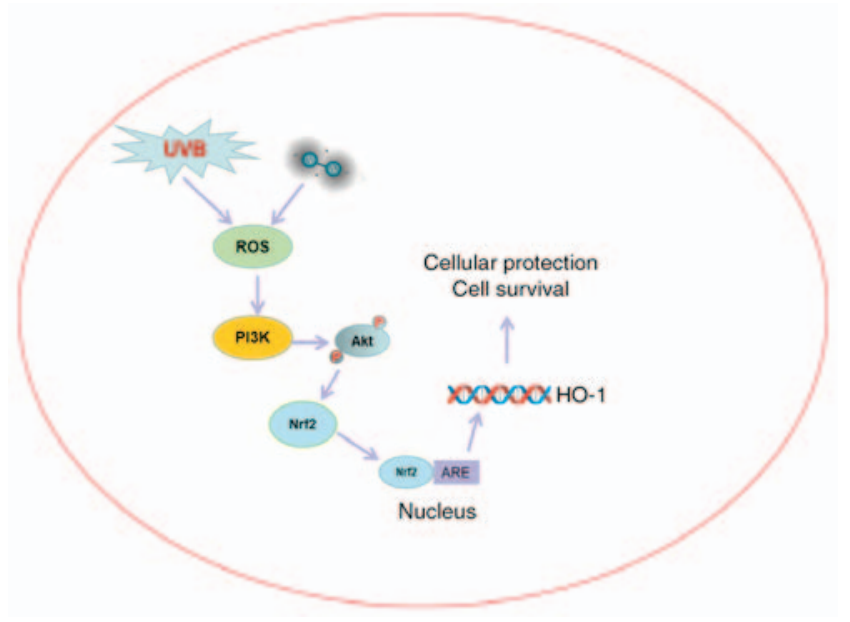

Figure 7. Schematic representation of the molecular mechanisms in cells in response to UVB radiation and the preventive effect of hydrogen. ROS, reactive oxygen species; PI3K, phosphoinositide-3 kinase; P, phosphate; HO-1, heme oxygenase 1; Nrf2, nuclear factor erythroid-2-related factor 2; ARE, antioxidant response element.

suggested that the protective effect of hydrogen was mediated by the activation of the PI3K/Akt pathway.

Hydrogen exerts its anti-oxidative effects via the PI3K/Akt pathway in UVB-irradiated HaCaT cells. The present study further investigated whether the effects of hydrogen to decrease ROS and its oxidative products occurred via PI3K/Akt. Compared with the UVB group, ROS, 8 -iso PGF2 $\alpha$ and MDA release were reduced in the $\mathrm{UVB}+\mathrm{H}_{2}$ group, but LY294002 abolished the anti-oxidant effect of hydrogen $(\mathrm{P}<0.05$; Fig. 5A-C). These results indicated that hydrogen exerted its anti-oxidative effects in UVB-irradiated HaCaT cells through the activation of the PI3K/Akt pathway.

Hydrogen activates Nrf2 expression through the PI3K/Akt pathway. To explore whether hydrogen stimulates the increase in Nrf2 protein via PI3K/Akt, the expression of the upstream proteins PI3K and Akt had been assessed (Fig. 3); furthermore, LY294002 was applied to assess the involvement of the PI3K/Akt pathway in the effects of hydrogen on the Nrf2 pathway. The results indicated that LY294002 inhibited Nrf2 translocation into the nucleus and reduced HO-1 and p-Akt levels in the $\mathrm{UVB}+\mathrm{H}_{2}+\mathrm{LY} 294003$ group compared with those in the $\mathrm{UVB}+\mathrm{H}_{2}$ group $(\mathrm{P}<0.05$; Fig. $6 \mathrm{~A}-\mathrm{D})$.

\section{Discussion}

As a potent environmental risk factors, exposure to UV irradiation is involved in the pathogenesis of several skin diseases (38). Of note, UVB primarily acts on the epidermal basal cell layer, whereas UVB may directly cause pathological changes of the skin. The present study focused on the effects of hydrogen, a newly identified gaseous signaling molecule, on $\mathrm{HaCaT}$ cells treated with UVB radiation. The results indicated that UVB radiation increased cell toxicity, reduced $\mathrm{HaCaT}$ cell proliferation, exacerbated oxidative stress injury by increasing the levels of ROS, 8 -iso PGF $2 \alpha$ and MDA, and upregulated 
the protein levels of Nrf2, HO-1, p-PI3K and p-Akt. However, hydrogen reduced the UVB radiation-induced cytotoxicity, inhibition of cell proliferation, and release of ROS, 8-iso PGF $2 \alpha$ and MDA in HaCaT cells, and further increased the levels of Nrf2, HO-1, p-PI3K and p-Akt, which were all upregulated via the PI3K-Akt signaling pathway.

The depletion of the ozone layer markedly aggravates the intensity of UVB radiation on the surface of the earth. Although UVB has certain beneficial functions, including its involvement in vitamin D synthesis, excessive exposure to UVB radiation causes various undesirable effects, including premature aging, solar erythema, cell damage and even skin cancer (39). Excessive generation of ROS from exposure of the skin to UVB may not be compensated by the anti-oxidant defense capacity of the skin, which leads to oxidative stress and oxidative photodamage to the skin $(40,41)$. Imbalances between the production and scavenging of ROS may stimulate the secretion of 8-iso PGF from HaCaT cells, which is a specific index of lipid peroxidation (42). As an end product of peroxidation, MDA damages cell membranes and indirectly indicates the extent of oxidative stress (43). Therefore, these biomarkers were assessed in the present study to reflect oxidative stress in $\mathrm{HaCaT}$ cells, and the results indicated that UVB radiation induced oxidative stress injury in $\mathrm{HaCaT}$ cells with increased levels of 8-iso PGF and MDA.

However, hydrogen, an anti-oxidant gas, was reported to inhibit oxidative processes associated with numerous diseases. For instance, in 2007, Ohsawa et al (44) indicated that hydrogen reduced the oxidative stress damage induced by focal ischemia and reperfusion of the brain by selectively reducing hydroxyl radicals and peroxynitrite (another cytotoxic ROS) in vitro. Also, hydrogen attenuated acute kidney injury induced by pacreatitis through inhibition of ROS in patients (45). In the present study, to verify whether hydrogen was able to protect $\mathrm{HaCaT}$ cells from UVB radiation-induced oxidative stress injury, cells were treated with hydrogen-saturated medium, which was revealed to obviously ameliorate cell toxicity and inhibition of cell proliferation, and reduce the production of ROS, 8-iso PGF and MDA induced by UVB radiation. These results indicated that hydrogen has a protective effect on $\mathrm{HaCaT}$ cells exposed to UVB radiation.

Although ROS were initially described as a disruptive factor, it is now known to be involved in regulating cell signaling, immune functions and gene transcription (46). ROS are associated with signaling that may activate transcription factor Nrf2 (47). In addition, as a master regulator of anti-oxidant enzymes and cellular stress resistance, Nrf2 is associated with UV-induced skin cell damage (20,48-51). After cell damage through exposure to UVB, Nrf2 translocates from the cytoplasm into the nucleus to combine with AREs, activate downstream cytoprotective genes and upregulate the antioxidant enzyme HO-1 to counteract UVB-mediated cellular injury (19). In addition, the present study indicated that $1-50 \mathrm{~mJ} / \mathrm{cm}^{2} \mathrm{UVB}$ stimulated HaCaT cells to produce excessive amounts of ROS. Subsequently, Nrf2 was presumably separated from Keap1 and released into the cytoplasm, after which $\mathrm{Nrf} 2$ translocated into the nucleus and activated the expression of the downstream HO-1 gene, resulting in the protection of $\mathrm{HaCaT}$ cells from the harms induced by excessive UVB radiation. These results were consistent with those of a previous study (52). Of note, the therapeutic effects of hydrogen in alleviating oxidative stress in numerous diseases, including dermatitis, neurodegenerative diseases and ischemia-reperfusion, are mediated via several important signaling pathways (53). The protective effect of hydrogen has been associated with the Nrf2 signaling pathway in different diseases models (54-56). A previous study by our group indicated that molecular hydrogen protected mice from polymicrobial sepsis via the Nrf2/HO-1 signaling pathway (27). The present study indicated that hydrogen stimulated UVB radiation-induced $\mathrm{Nrf} 2$ translocation and $\mathrm{HO}-1$ expression to further protect cells against oxidative stress injury.

Furthermore, Nguyen et al (57) demonstrated that the activation of Nrf2-mediated $\mathrm{HO}-1$ production via the $\mathrm{PI} 3 \mathrm{~K} / \mathrm{Akt}$ and/or protein kinase $\mathrm{C}$ pathways has a critical role in the protective effect of caffeoylserotonin against oxidative stress-induced keratinocyte cell death. Of note, PI3K/Akt signaling is important for boosting cell proliferation and migration in various diseases and cancer types (58-61). In addition, ROS not only activated the Nrf2 pathway directly to amplify its downstream signaling, but also positively regulated PI3K activation and expression of its target genes, which was activated through autophosphorylation (62). Once PI3K is phosphorylated, it activates Akt, which is a key downstream molecule of PI3K. The PI3K/Akt signaling pathway is associated with the Nrf2 pathway in human keratinocytes $(57,63,64)$. The PI3K/Akt-mediated Nrf2/HO-1 signaling pathway was also reported to have a critical role in the 7,8-dihydroxyflavone-mediated protection of human keratinocytes from oxidative stress-induced cell damage (63). In addition, Li et al (65) demonstrated that hydrogen ameliorated liver ischemia/reperfusion injury, which featured marked oxidative stress injury, through inhibition of the PI3K/Akt signaling pathway. Collectively, these aboveme ntioned studies prompted us to hypothesize that PI3K/Akt signaling is involved in the protective effects of hydrogen on $\mathrm{HaCaT}$ cells that were exposed to excessive UVB radiation. An inhibitor of PI3K was then employed to confirm this notion. The results of the present study indicated that UVB radiation stimulated the phosphorylation of PI3K and Akt in $\mathrm{HaCaT}$ cells, while the hydrogen treatment obviously further increased the p-PI3K and p-Akt levels after UVB irradiation. Furthermore, the specific PI3K inhibitor LY294002 partially reversed the effect of hydrogen in attenuating the cytotoxicity, inhibition of cell proliferation and production of ROS, 8-iso PGF $2 \alpha$ and MDA stimulated by UVB. The results of the present study indicated that hydrogen may serve a role in amplifying the protective response to UVB-induced damage, which may be inhibited by LY294002. Hydrogen may have amplified the effects of irradiation on the expression PI3K in response to radiation; however, it may be difficult to distinguish between the effects of hydrogen and the cellular response to radiation. When PI3K was inhibited, Nrf2 translocation to eliminate ROS was suppressed, so that ROS generation accumulated to decrease the cellular function and proliferation. Thus, a negative feedback loop was then formed. These results supported the conclusion that hydrogen exerted protective effects against UVB-induced cytotoxicity and reduction of proliferation of $\mathrm{HaCaT}$ cells by quenching excessive ROS, leading to the inhibition of the production of 8 -iso PGF2 $\alpha$ and MDA, as well as by promotion of nuclear translocation of Nrf2 and HO-1 expression through the PI3K-Akt signaling pathway. 
In conclusion, the present study provided evidence that UVB radiation has cytotoxic effects and inhibits cell proliferation, and stimulates ROS production and the generation of their oxidation products, and that PIK3/Akt and Nrf2/HO-1 contribute to the oxidative stress response of HaCaT cells induced by UVB radiation. Furthermore, hydrogen exerts a protective effect by increasing Nrf2/HO-1 levels via the PI3K-Akt signaling pathway in UVB-irradiated $\mathrm{HaCaT}$ cells (Fig. 7). Although $\mathrm{PI} 3 \mathrm{~K} / \mathrm{Akt}$ was demonstrated to be involved in the protective effect of hydrogen in UVB-induced oxidative stress injury, further investigations of the specific mechanisms are required for future applications of hydrogen in the clinic.

\section{Acknowledgements}

Not applicable.

\section{Funding}

The present study was supported by the grants from the National Natural Science Foundation of China (grant no. 81601667).

\section{Availability of data and materials}

The datasets used and/or analyzed during the current study are available from the corresponding author on reasonable request.

\section{Authors' contributions}

ZBX and ZZS designed the research. ZBX, ZZS and MXY conducted the experimental protocols in the present study. $\mathrm{CHG}$ acquired analytical reagents and tools. Data analysis was performed by CHG. ZBX and ZZS prepared the manuscript.

\section{Ethics approval and consent to participate}

Not applicable.

\section{Consent for publication}

Not applicable.

\section{Competing interests}

The authors declare that they have no competing interests.

\section{References}

1. Sharma SD and Katiyar SK: Dietary grape seed proanthocyanidins inhibit UVB-induced cyclooxygenase-2 expression and other inflammatory mediators in UVB-exposed skin and skin tumors of SKH-1 hairless mice. Pharm Res 27: 1092-1102, 2010.

2. Helfrich YR, Sachs DL and Voorhees JJ: Overview of skin aging and photoaging. Dermatol Nurs 20: 177-183; quiz 184, 2008.

3. Bernstein EF, Chen YQ, Tamai K, Shepley KJ, Resnik KS, Zhang H, Tuan R, Mauviel A and Uitto J: Enhanced elastin and fibrillin gene expression in chronically photodamaged skin. J Invest Dermatol 103: 182-186, 1994.

4. Avila Acevedo JG, Espinosa González AM, De Maria y Campos DM, Benitez Flores Jdel C, Hernández Delgado T, Flores Maya S, Campos Contreras J, Muñoz López JL and García Bores AM: Photoprotection of Buddleja cordata extract against UVB-induced skin damage in SKH-1 hairless mice. BMC Complement Altern Med 14: 281, 2014.
5. de Gruijl FR: Photocarcinogenesis: UVA vs UVB. Methods Enzymol 319: 359-366, 2000.

6. Katiyar SK: Silymarin and skin cancer prevention: Antiinflammatory, antioxidant and immunomodulatory effects (Review). Int J Oncol 26: 169-176, 2005.

7. Sime S and Reeve VE: Protection from inflammation, immunosuppression and carcinogenesis induced by UV radiation in mice by topical Pycnogenol. Photochem Photobiol 79: 193-198, 2004.

8. Narayanan DL, Saladi RN and Fox JL: Ultraviolet radiation and skin cancer. Int J Dermatol 49: 978-986, 2010.

9. Lee SJ and Park JW: Enhancement of UVB radiation-mediated apoptosis by knockdown of cytosolic NADP+-dependent isocitrate dehydrogenase in HaCaT cells. BMB Rep 47: 209-214, 2014.

10. Lee KO, Kim SN and Kim YC: Anti-wrinkle effects of water extracts of teas in hairless mouse. Toxicol Res 30: 283-289, 2014.

11. Palmer DM and Kitchin JS: Oxidative damage, skin aging, antioxidants and a novel antioxidant rating system. J Drugs Dermatol 9: 11-15, 2010.

12. Sklar LR, Almutawa F, Lim HW and Hamzavi I: Effects of ultraviolet radiation, visible light, and infrared radiation on erythema and pigmentation: A review. Photochem Photobiol Sci 12: 54-64, 2013.

13. Vayalil PK, Mittal A, Hara Y, Elmets CA and Katiyar SK: Green tea polyphenols prevent ultraviolet light-induced oxidative damage and matrix metalloproteinases expression in mouse skin. J Invest Dermatol 122: 1480-1487, 2004.

14. Verschooten L, Claerhout S, Van Laethem A, Agostinis P and Garmyn M: New strategies of photoprotection. Photochem Photobiol 82: 1016-1023, 2006.

15. Piao MJ, Kang KA, Kim KC, Chae S, Kim GO, Shin T, Kim HS and Hyun JW: Diphlorethohydroxycarmalol attenuated cell damage against UVB radiation via enhancing antioxidant effects and absorbing UVB ray in human HaCaT keratinocytes. Environ Toxicol Pharmacol 36: 680-688, 2013.

16. Tao S, Justiniano R, Zhang DD and Wondrak GT: The Nrf2-inducers tanshinone I and dihydrotanshinone protect human skin cells and reconstructed human skin against solar simulated UV. Redox Biol 1: 532-541, 2013.

17. Schäfer M, Dütsch S, auf dem Keller U, Navid F, Schwarz A, Johnson DA, Johnson JA and Werner S: Nrf2 establishes a glutathione-mediated gradient of UVB cytoprotection in the epidermis. Genes Dev 24: 1045-1058, 2010.

18. Saw CL, Huang MT, Liu Y, Khor TO, Conney AH and Kong AN: Impact of Nrf2 on UVB-induced skin inflammation/photoprotection and photoprotective effect of sulforaphane. Mol Carcinog 50: 479-486, 2011.

19. Kannan S and Jaiswal AK: Low and high dose UVB regulation of transcription factor NF-E2-related factor 2. Cancer Res 66: 8421-8429, 2006.

20. Zhong J and Li L: Skin-derived precursors against UVB-induced apoptosis via Bcl-2 and Nrf2 upregulation. Biomed Res Int 2016: 6894743, 2016.

21. Wang L, Chen Y, Sternberg P and Cai J: Essential roles of the PI3 kinase/Akt pathway in regulating Nrf2-dependent antioxidant functions in the RPE. Invest Ophthalmol Vis Sci 49: 1671-1678, 2008.

22. Lee YJ, Jeong HY, Kim YB, Lee YJ, Won SY, Shim JH, Cho MK, Nam HS and Lee SH: Reactive oxygen species and PI3K/Akt signaling play key roles in the induction of Nrf2-driven heme oxygenase-1 expression in sulforaphane-treated human mesothelioma MSTO-211H cells. Food Chem Toxicol 50: 116-123, 2012.

23. Xu Y, Duan C, Kuang Z, Hao Y, Jeffries JL and Lau GW: Pseudomonas aeruginosa pyocyanin activates NRF2-ARE-mediated transcriptional response via the ROS-EGFR-PI3K-AKT/MEK-ERK MAP kinase signaling in pulmonary epithelial cells. PLoS One 8: e72528, 2013.

24. Li KR, Yang SQ, Gong YQ, Yang H, Li XM, Zhao YX, Yao J, Jiang $Q$ and Cao C: $3 \mathrm{H}-1,2$-dithiole-3-thione protects retinal pigment epithelium cells against Ultra-violet radiation via activation of Akt-mTORC1-dependent Nrf2-HO-1 signaling. Sci Rep 6: 25525, 2016.

25. Zhang H, Liu YY, Jiang Q, Li KR, Zhao YX, Cao C and Yao J: Salvianolic acid A protects RPE cells against oxidative stress through activation of Nrf2/HO-1 signaling. Free Radic Biol Med 69: 219-228, 2014.

26. Rodriguez KJ, Wong HK, Oddos T, Southall M, Frei B and Kaur S: A purified feverfew extract protects from oxidative damage by inducing DNA repair in skin cells via a PI3-kinase-dependent Nrf2/ARE pathway. J Dermatol Sci 72: 304-310, 2013. 
27. Chen H, Xie K, Han H, Li Y, Liu L, Yang T and Yu Y: Molecular hydrogen protects mice against polymicrobial sepsis by ameliorating endothelial dysfunction via an $\mathrm{Nrf} 2 / \mathrm{HO}-1$ signaling pathway. Int Immunopharmacol 28: 643-654, 2015.

28. Li DZ, Zhang QX, Dong XX, Li HD and Ma X: Treatment with hydrogen molecules prevents RANKL-induced osteoclast differentiation associated with inhibition of ROS formation and inactivation of MAPK, AKT and NF-kappa B pathways in murine RAW264.7 cells. J Bone Miner Metab 32: 494-504, 2014.

29. Hong Y, Shao A, Wang J, Chen S, Wu H, McBride DW, Wu Q, Sun $\mathrm{X}$ and Zhang J: Neuroprotective effect of hydrogen-rich saline against neurologic damage and apoptosis in early brain injury following subarachnoid hemorrhage: Possible role of the Akt/GSK3beta signaling pathway. PLoS One 9: e96212, 2014.

30. Yu P, Wang Z, Sun X, Chen X, Zeng S, Chen L and Li S: Hydrogen-rich medium protects human skin fibroblasts from high glucose or mannitol induced oxidative damage. Biochem Biophys Res Commun 409: 350-355, 2011.

31. Mei K, Zhao S, Qian L, Li B, Ni J and Cai J: Hydrogen protects rats from dermatitis caused by local radiation. J Dermatolog Treat 25: 182-188, 2014

32. Ishibashi T, Ichikawa M, Sato B, Shibata S, Hara Y, Naritomi Y, Okazaki K, Nakashima Y, Iwamoto Y, Koyanagi S, et al: Improvement of psoriasis-associated arthritis and skin lesions by treatment with molecular hydrogen: A report of three cases. Mol Med Rep 12: 2757-2764, 2015.

33. Meng CY: Effect of hydrogen-rich saline on allergic contact dermatitis in mice. Chin J Dermatol 46: 275-278, 2013.

34. Zhang BX: Hydrogen-rich liquid down-regulates the expressions of inflammatory facmrs by ultraviolet B-induced human HaCaT keratinocytes through the autophagy pathway. Chin J Dermatol 49: 123-127, 2016 (In Chinese).

35. Xing WB, Fu GJ, Ye WJ, Qin LY, Chen HG, Meng XY and Meng CY: Effects of hydrogen on ultraviolet B-induced oxidative damage to skin fibroblasts. Chin J Dermatol 46: 424-426, 2013.

36. Chen HG, Xie KL, Han HZ, Wang WN, Liu DQ, Wang GL and Yu YH: Heme oxygenase-1 mediates the anti-inflammatory effect of molecular hydrogen in LPS-stimulated RAW 264.7 macrophages. Int J Surg 11: 1060-1066, 2013

37. Tongqiang L, Shaopeng L, Xiaofang Y, Nana S, Xialian X, Jiachang $\mathrm{H}$, Ting Z and Xiaoqiang D: Salvianolic acid B prevents iodinated contrast media-induced acute renal injury in rats via the PI3K/Akt/Nrf2 pathway. Oxid Med Cell Longev 2016: 7079487, 2016.

38. Song JL and Gao Y: Protective effects of lindera coreana on UVB-induced oxidative stress in human HaCaT keratinocytes Iran J Pharm Res 13: 1369-1378, 2014.

39. Drouin R and Therrien JP: UVB-induced cyclobutane pyrimidine dimer frequency correlates with skin cancer mutational hotspots in p53. Photochem Photobiol 66: 719-726, 1997.

40. Podhaisky HP, Riemschneider S and Wohlrab W: UV light and oxidative damage of the skin. Pharmazie 57: 30-33, 2002.

41. Sander CS, Chang H, Salzmann S, Müller CS, Ekanayake-Mudiyanselage S, Elsner P and Thiele JJ: Photoaging is associated with protein oxidation in human skin in vivo. J Invest Dermatol 118: 618-625, 2002.

42. Beauchamp MC, Letendre E and Renier G: Macrophage lipoprotein lipase expression is increased in patients with heterozygous familial hypercholesterolemia. J Lipid Res 43: 215-222, 2002.

43. Bai K, Xu W, Zhang J, Kou T, Niu Y, Wan X, Zhang L, Wang C and Wang T: Assessment of free radical scavenging activity of dimethylglycine sodium salt and its role in providing protection against lipopolysaccharide-induced oxidative stress in mice. PLoS One 11: e155393, 2016.

44. Ohsawa I, Ishikawa M, Takahashi K, Watanabe M, Nishimaki K, Yamagata K, Katsura K, Katayama Y, Asoh S and Ohta S: Hydrogen acts as a therapeutic antioxidant by selectively reducing cytotoxic oxygen radicals. Nat Med 13: 688-694, 2007.

45. Shi Q, Liao KS, Zhao KL, Wang WX, Zuo T, Deng WH, Chen C, Yu J, Guo WY, He XB, et al: Hydrogen-rich saline attenuates acute renal injury in sodium taurocholate-induced severe acute pancreatitis by inhibiting ROS and NF-kB pathway. Mediators Inflamm 2015: 685043, 2015.

46. Done AJ and Traustadóttir T: Nrf2 mediates redox adaptations to exercise. Redox Biol 10: 191-199, 2016.

47. Shih PH, Yeh CT and Yen GC: Anthocyanins induce the activation of phase II enzymes through the antioxidant response element pathway against oxidative stress-induced apoptosis J Agric Food Chem 55: 9427-9435, 2007.
48. Uchi H, Yasumatsu M, Morino-Koga S, Mitoma C and Furue M: Inhibition of aryl hydrocarbon receptor signaling and induction of NRF2-mediated antioxidant activity by cinnamaldehyde in human keratinocytes. J Dermatol Sci 85: 36-43, 2017.

49. Sun Z, Park SY, Hwang E, Zhang M, Seo SA, Lin P and Yi TH: Thymus vulgaris alleviates UVB irradiation induced skin damage via inhibition of MAPK/AP-1 and activation of Nrf2-ARE antioxidant system. J Cell Mol Med 21: 336-348, 2017.

50. Shan Y, Wei Z, Tao L, Wang S, Zhang F, Shen C, Wu H, Liu Z, Zhu P, Wang A, et al: Prophylaxis of Diallyl disulfide on skin carcinogenic model via p21-dependent Nrf2 stabilization. Sci Rep 6: 35676, 2016.

51. Ji C, Huang JW, Xu QY, Zhang J, Lin MT, Tu Y, He L, Bi ZG and Cheng B: Gremlin inhibits UV-induced skin cell damages via activating VEGFR2-Nrf2 signaling. Oncotarget 7: 84748-84757, 2016.

52. Yuan XY, Pang XW, Zhang GQ and Guo JY: Salidroside's protection against UVB-mediated oxidative damage and apoptosis is associated with the upregulation of Nrf2 expression. Photomed Laser Surg 35: 49-56, 2017.

53. Xie K, Liu L, Yu Y and Wang G: Hydrogen gas presents a promising therapeutic strategy for sepsis. Biomed Res Int 2014: 807635, 2014

54. Diao M, Zhang S, Wu L, Huan L, Huang F, Cui Y and Lin Z: Hydrogen gas inhalation attenuates seawater instillation-induced acute lung injury via the Nrf2 pathway in rabbits. Inflammation 39: 2029-2039, 2016

55. Tamaki N, Orihuela-Campos RC, Fukui $\mathrm{M}$ and Ito $\mathrm{HO}$ Hydrogen-rich water intake accelerates oral palatal wound healing via activation of the Nrf2/antioxidant defense pathways in a rat model. Oxid Med Cell Longev 2016: 5679040, 2016.

56. Yu J, Zhang W, Zhang R, Jiang G, Tang H, Ruan X, Ren P and Lu B: Molecular hydrogen attenuates hypoxia/reoxygenation injury of intrahepatic cholangiocytes by activating Nrf2 expression. Toxicol Lett 238: 11-19, 2015.

57. Nguyen CN, Kim HE and Lee SG: Caffeoylserotonin protects human keratinocyte $\mathrm{HaCaT}$ cells against $\mathrm{H}_{2} \mathrm{O}_{2}$-induced oxidative stress and apoptosis through upregulation of HO-1 expression via activation of the PI3K/Akt/Nrf2 pathway. Phytother Res 27: 1810-1818, 2013.

58. Hermida MA, Dinesh Kumar J and Leslie NR: GSK3 and its interactions with the PI3K/AKT/mTOR signalling network. Adv Biol Regul 65: 5-15, 2017.

59. Follo MY, Manzoli L, Poli A, McCubrey JA and Cocco L: PLC and PI3K/Akt/mTOR signalling in disease and cancer. Adv Biol Regul 57: 10-16, 2015.

60. Jhanwar-Uniyal M, Amin AG, Cooper JB, Das K, Schmidt MH and Murali R: Discrete signaling mechanisms of mTORC1 and mTORC2: Connected yet apart in cellular and molecular aspects. Adv Biol Regul 64: 39-48, 2017.

61. Ricciardi MR, Mirabilii S, Licchetta R, Piedimonte M and Tafuri A: Targeting the Akt, GSK-3, Bcl-2 axis in acute myeloid leukemia. Adv Biol Regul 65: 36-58, 2017.

62. Jayasooriya RG, Lee KT, Choi YH, Moon SK, Kim WJ and Kim GY: Antagonistic effects of acetylshikonin on LPS-induced $\mathrm{NO}$ and $\mathrm{PGE}_{2}$ production in BV2 microglial cells via inhibition of ROS/PI3K/Akt-mediated NF- $\mathrm{KB}$ signaling and activation of Nrf2-dependent HO-1. In Vitro Cell Dev Biol Anim 51: 975-986, 2015.

63. Ryu MJ, Kang KA, Piao MJ, Kim KC, Zheng J, Yao CW, Cha JW, Chung HS, Kim SC, Jung E, et al: 7,8-Dihydroxyflavone protects human keratinocytes against oxidative stress-induced cell damage via the ERK and PI3K/Akt-mediated Nrf2/HO-1 signaling pathways. Int J Mol Med 33: 964-970, 2014.

64. Seo SH and Jeong GS: Fisetin inhibits TNF- $\alpha$-induced inflammatory action and hydrogen peroxide-induced oxidative damage in human keratinocyte $\mathrm{HaCaT}$ cells through PI3K/AKT/Nrf-2-mediated heme oxygenase-1 expression. Int Immunopharmacol 29: 246-253, 2015.

65. Li H, Chen O, Ye Z, Zhang R, Hu H, Zhang N, Huang J, Liu W and Sun X: Inhalation of high concentrations of hydrogen ameliorates liver ischemia/reperfusion injury through A2A receptor mediated PI3K-Akt pathway. Biochem Pharmacol 130: 83-92, 2017. 